\title{
Differential effects of HTO and OBT ingestion or external irradiation on iron metabolism
}

\author{
Jean-Marc Bertho ${ }^{1, *}$, Dimitri Kereselidze ${ }^{1}$, Line Manens ${ }^{1}$, Cécile Culeux ${ }^{1}$, Joel Surette $^{2}$, \\ Melinda Blimke ${ }^{2}$, Linsday Bertrand ${ }^{2}$,Heather Wyatt $^{2}$, Maamar Souidi ${ }^{1}$, Nick Priest ${ }^{2}$, Jean- \\ René Jourdain ${ }^{1}$. \\ ${ }^{1}$ IRSN, PSE-SAN/SESANE, LRTOX, BP 17, 92262 Fontenay aux roses cedex, France \\ ${ }^{2}$ Canadian Nuclear Laboratories Ltd, Chalk River, ON, Canada.
}

\section{Introduction}

Environmental exposure to tritium is mainly due to tritium releases by nuclear reactors. This exposure may increase in future years due to potential increased releases by the ITER reactor. However concerns exist about the reliability of the current ICRP recommendations. In fact, the current regulatory level is set at $7000 \mathrm{~Bq} . \mathrm{L}^{-1}$ in Canada, WHO recommendation is set at $10 \mathrm{kBq} \cdot \mathrm{L}^{-1}$ and French regulatory level is set at $100 \mathrm{~Bq} \cdot \mathrm{L}^{-1}$. As a result, a collaborative project was developed between IRSN and CNL to study various aspects of the toxicity of tritium.

\section{Material and methods}

In this project male $\mathrm{C} 57 \mathrm{BL} / 6 \mathrm{~J}$ mice were exposed to a chronic contamination through drinking water with three different concentrations of tritium (HTO), or tritiated amino acids (TAA) or low dose external gamma radiation (1.3 and $31.2 \mu \mathrm{Gy} . \mathrm{h}-1)$, equivalent to tritium exposure, for either 1 or 8 months. Drinking water concentrations of tritium were $10 \mathrm{kBq} . \mathrm{L}^{-}$ 1, $1 \mathrm{MBq} \cdot \mathrm{L}^{-1}$ and $20 \mathrm{MBq} \cdot \mathrm{L}^{-1}$. The lowest concentration used in this study is relevant to WHO recommendations. At 1 and 8 months of exposure, blood, spleen, liver, kidney and intestine were harvested and subjected to various analyses including blood cell count and differential, measurement of biochemical parameters in the plasma, relevant genes expression quantification and relevant immunohistological analyses.

\section{Results}

Results of blood cell count analyses indicated slight but significant decrease in RBC at 1 month of exposure with TAA which was followed at 8 months by an increase in the size of RBC. These changes were not observed with HTO exposure. These results suggest the appearance of an anaemia at 1 month of exposure with TAA, which was compensated by an increased half-life of red blood cells at 8 months of exposure. A concomitant decrease in serum iron concentration at 8 months of exposure in TAA-exposed animals was observed

* Corresponding author: jean-marc.bertho@irsn.fr 
with a dose dependent effect suggesting that an iron deficit was at the origin of this anaemia. In fact, the increased life span of red blood cells is a classically described compensation mechanism for a mild iron deficit. Such an iron-dependent anaemia may be due to either a central defect of haematopoiesis, a defect in EPO production by the kidneys, a defect in the capture of RBC in the spleen or a defect in iron metabolism. Results of gene expression analysis, histological analysis and cytokine measurement allowed excluding the three first hypotheses. However, a decrease in ERFE expression in the liver at one month and a decrease in transferrin light chain expression in intestine, both at 1 month and 8 months of exposure were evidenced. ERFE is implicated in a regulation loop of iron concentration in the blood through the inhibition of Hepcidin, and transferrin is implicated into the intracellular iron transport. Interestingly, a change in the pattern of histological localization of DMT1 was observed in the intestinal villi, suggesting that the iron transport through the intestinal epithelium is the key parameter that may explain these results. Work is currently in progress to confirm these results.

\section{Conclusions}

Overall these results demonstrate a differential effect of HTO and OBT at the same level of exposure. In parallel, no effect was observed in animals gamma irradiated at a low dose rate equivalent to the dose rate due to incorporated tritium. This suggests that deterministic effects may appear during chronic ingestion of tritium and that these effects may be significantly more important with an OBT form of tritium as compared to HTO. This may be explained by the integration of TAA in the general metabolism, and more specifically in the metabolism of intestinal epithelial cells. These results also suggest that the effect of TAA might be dependent upon the physiology of the amino acids used in this study, i.e. on the specific pattern of exposure to tritium beta rays according to the micro anatomic location of these amino acids. These results also suggest that the biological effects of other forms of OBT, e.g. tritiated sugars or tritiated lipids, might be very different. The implication of these results for radioprotection remains to be defined. 